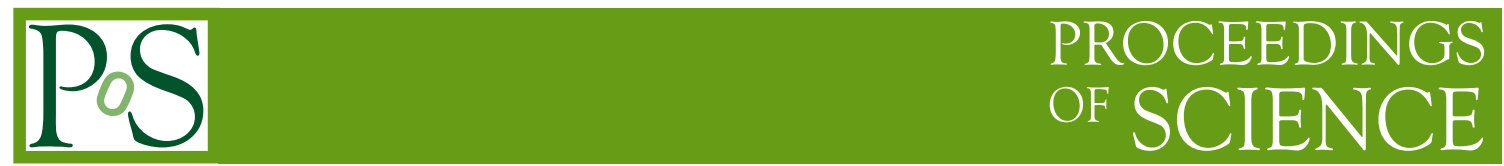

\title{
Effective thermal models from the lattice
}

\section{Sourendu Gupta*}

Tata Institute of Fundamental Research

E-mail: sgupta@theory.tifr.res.in

\section{Rishi Sharma}

Tata Institute of Fundamental Research

E-mail: rishidtheory.tifr.res.in

We build effective models of the thermodynamics of QCD and match the couplings using a few measurements from the lattice. We discuss further predictions from the models, including corners where the lattice cannot reach yet.

Critical Point and Onset of Deconfinement - CPOD2017

7-11 August, 2017

The Wang Center, Stony Brook University, Stony Brook, NY

\footnotetext{
* Speaker.
} 
It seems possible to understand very low temperature QCD in terms of a pion gas, and very high temperature QCD in terms of a weakly-coupled quark-gluon gas. It seems likely that the two pictures become more and more complicated as they are extended towards the cross over between these regimes. The QCD cross over region is not understood except through lattice computations. This is part of a series of works aimed at understanding some aspects of the physics here. An earlier part was reported in [1], and that, as well as the material in this talk is included in [2].

We can start by restricting our attention to a small region around a temperature $T_{0}$, and asking whether we can understand amplitudes with all momenta $p \ll T_{0}$. If we are interested only in flavoured amplitudes, then it may be sufficient to write an effective field theory model in terms of quarks. Keeping all terms up to dimension 6 which are invariant under spatial rotations, and the flavour symmetry of $N_{f}=2 \mathrm{QCD}$, with $\mathrm{C}, \mathrm{P}$ and T invariance gives the Lagrangian density

$$
\begin{aligned}
L= & d^{3} T_{0} \bar{\psi} \psi+\bar{\psi} \partial_{4} \psi+d^{4} \bar{\psi} \chi_{i} \psi+L_{6} \text { where } \\
L_{6}= & +\frac{d^{61}}{T_{0}^{2}}\left[(\bar{\psi} \psi)^{2}+\left(\bar{\psi} i \gamma_{5} \tau^{a} \psi\right)^{2}\right]+\frac{d^{62}}{T_{0}^{2}}\left[\left(\bar{\psi} \tau^{a} \psi\right)^{2}+\left(\bar{\psi} i \gamma_{5} \psi\right)^{2}\right] \\
& +\frac{d^{63}}{T_{0}^{2}}\left(\bar{\psi} \gamma_{4} \psi\right)^{2}+\frac{d^{64}}{T_{0}^{2}}\left(\bar{\psi} i \gamma_{i} \psi\right)^{2}+\frac{d^{65}}{T_{0}^{2}}\left(\bar{\psi} \gamma_{5} \gamma_{4} \psi\right)^{2}+\frac{d^{66}}{T_{0}^{2}}\left(\bar{\psi} i \gamma_{5} \gamma_{i} \psi\right)^{2} \\
& +\frac{d^{67}}{T_{0}^{2}}\left[\left(\bar{\psi} \gamma_{4} \tau^{a} \psi\right)^{2}+\left(\bar{\psi} \gamma_{5} \gamma_{4} \tau^{a} \psi\right)^{2}\right]+\frac{d^{68}}{T_{0}^{2}}\left[\left(\bar{\psi} i \gamma_{i} \tau^{a} \psi\right)^{2}+\left(\bar{\psi} i \gamma_{5} \gamma_{i} \tau^{a} \psi\right)^{2}\right] \\
& +\frac{d^{69}}{T_{0}^{2}}\left[\left(\bar{\psi} i S_{i 4} \psi\right)^{2}+\left(\bar{\psi} S_{i j} \tau^{a} \psi\right)^{2}\right]+\frac{d^{60}}{T_{0}^{2}}\left[\left(\bar{\psi} i S_{i 4} \tau^{a} \psi\right)^{2}+\left(\bar{\psi} S_{i j} \psi\right)^{2}\right]
\end{aligned}
$$

The iso-spin generators are $\tau^{a}$, and conventions for the Euclidean Dirac matrices are given in [2]. Even though this has many more couplings, the mean field theory is very similar to that of the NJL model [3]. One of the new ingredients is that there is a difference between pole and screening masses $[1,2]$. Including this, there turn out to be three independent couplings in the mean field theory. We find, as expected, a cross over between two phases: one with chiral symmetry broken and the other with the symmetry restored. We can choose $T_{0}$ to be the temperature at the critical point in the chiral limit.

In order to fix the parameters of the model, it turns out to be useful to first examine fluctuations around the mean-field theory in the symmetry broken low-temperature phase. As long as fluctuations in the magnitude of the chiral condensate can be neglected, it is the chiral angular fluctuations corresponding to pions which need to be examined. These can be parametrized as

$$
\psi \rightarrow \mathrm{e}^{i \pi^{a} \tau^{a} \gamma_{5} /(2 f)} \psi \quad \text { and } \quad \bar{\psi} \rightarrow \bar{\psi} \mathrm{e}^{i \pi^{a} \tau^{a} \gamma_{5} /(2 f)},
$$

where $\pi^{a}$ correspond to pion fields ( $a$ is an isospin index), and, in this context, $f$ is the finitetemperature analogue of the pion decay constant.

The pion effective action up to all fourth order terms can be written as

$$
L_{f}=\frac{c^{2} T_{0}^{2}}{2} \pi^{2}+\frac{1}{2}\left(\partial_{0} \pi\right)^{2}+\frac{c^{4}}{2}(\nabla \pi)^{2}+\frac{c^{41}}{8} \pi^{4} .
$$

All three dimensionless couplings, and the parameter $f$ in eq. (2) can be obtained by integrating out the fermions. We do this to one-loop order. This relates these 4 parameters above to three 
couplings in the fermion effective action. Additional information about the location of the cutoff is also used to tune the parameters of the fermion theory. As a result, only two of the parameters of the pion theory are independent; we choose these to be $c^{2}$ and $c^{4}$. In [4], a similar action was postulated with $c^{41}$ set to zero. There $c^{2} T_{0}^{2}$ was identified with the pion pole mass, and $c^{4}$ with the square of the pion "velocity" $u^{2}$.

This extremely simple model is fitted to a two-flavour lattice simulation [5]. Assuming that the parameters of the fermion effective action are independent of the temperature and quark mass in a small range of temperature around the cross over, one finds that the chiral critical point is at $T_{0}=170 \pm 6 \mathrm{MeV}$. This agrees with the preliminary estimate for $N_{f}=2$ given in [6]. There is a mismatch of notation between the lattice computation in [5] and the notation used in [2] which is explained in the latter. In the notation of the latter, the comparison of the effective model and the lattice is shown in Figure 1.
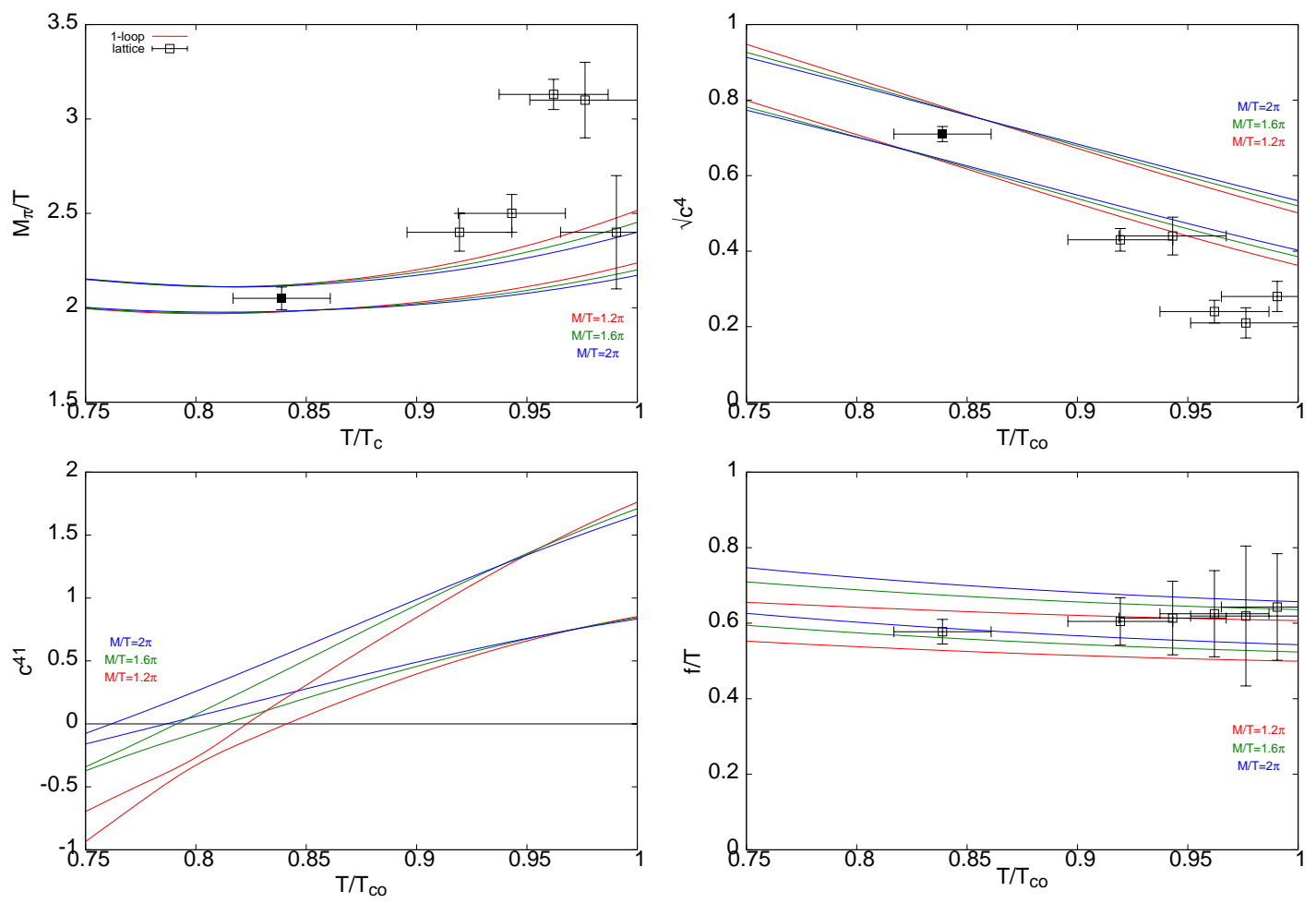

Figure 1: Comparison of the effective model with lattice measurements. The parameters are fixed by the pion screening mass, $M_{\pi}$, and $\sqrt{c^{4}}$ as measured at one temperature (shown with filled symbols). Everything else is a prediction of the model. There is an interesting rough match between the model and the results of the lattice computation. Future measurements of pion 4-point functions could yield $c^{41}$.

The pleasantly reasonable agreement between data and the effective theory shows that it may be possible to understand physics in the region of the QCD cross over, at least for very small momenta, in terms of few degrees of freedom. This encourages us to extend this computation to real time, a study whose results will be reported later. 


\section{References}

[1] S. Gupta and R. Sharma, Nucl. Phys. A 967 (2017) 716 [arXiv:1704.04495 [hep-ph]].

[2] S. Gupta and R. Sharma, arXiv:1710.05345 [hep-ph].

[3] S. P. Klevansky, Rev. Mod. Phys. 64 (1992) 649.

[4] D. T. Son and M. A. Stephanov, Phys. Rev. D 66 (2002) 076011 [hep-ph/0204226].

[5] B. B. Brandt, A. Francis, H. B. Meyer and D. Robaina, Phys. Rev. D 90 (2014) no.5, 054509 [arXiv:1406.5602 [hep-lat]].

[6] B. B. Brandt, A. Francis, H. B. Meyer, O. Philipsen and H. Wittig, PoS LATTICE 2013 (2014) 162 [arXiv:1310.8326 [hep-lat]]. 